\title{
L-Citrulline Attenuates Arrested Alveolar Growth and Pulmonary Hypertension in Oxygen-Induced Lung Injury in Newborn Rats
}

\author{
ARUL VADIVEL, JUDY L. ASCHNER, GLORIA J. REY-PARRA, JORDAN MAGARIK, HENG ZENG, MARSHALL SUMMAR, \\ FARAH EATON, AND BERNARD THÉBAUD
}

Department of Pediatrics [A.V., G.J.R.-P., F.E., B.T.], Women and Children's Health Research Institute, University of Alberta, Edmonton, Alberta T6G 2S2, Canada; Departments of Pediatrics [J.L.A., J.M., H.Z., M.S.] and Molecular Physiology and Biophysics [J.M., M.S.],

Vanderbilt University Medical Center, Nashville, Tennesse 37232

\begin{abstract}
Bronchopulmonary dysplasia (BPD) is characterized by arrested alveolar development and complicated by pulmonary hypertension (PH). NO promotes alveolar growth. Inhaled NO (iNO) ameliorates the BPD phenotype in experimental models and in some premature infants. Arginosuccinate synthetase (ASS) and arginosuccinate lyase (ASL) convert L-citrulline to L-arginine; L-citrulline is regenerated during NO synthesis from L-arginine. Plasma levels of these NO precursors are low in PH. We hypothesized that L-citrulline prevents experimental $\mathrm{O}_{2}$-induced BPD in newborn rats. Rat pups were assigned from birth through postnatal day (P) 14 to room air (RA), RA + L-citrulline, 95\% hyperoxia (BPD model), and $95 \% \mathrm{O}_{2}+\mathrm{L}$-citrulline. Rat pups exposed to hyperoxia had fewer and enlarged air spaces and decreased capillary density, mimicking human BPD. This was associated with decreased plasma L-arginine and L-citrulline concentrations on P7. L-Citrulline treatment significantly increased plasma Larginine and L-citrulline concentrations and increased ASL protein expression in hyperoxia. L-Citrulline preserved alveolar and vascular growth in $\mathrm{O}_{2}$-exposed pups and decreased pulmonary arterial medial wall thickness (MWT) and right ventricular hypertrophy (RVH). Increased lung arginase (ARG) activity in $\mathrm{O}_{2}$ exposed pups was reversed by L-citrulline treatment. L-Citrulline supplementation prevents hyperoxia-induced lung injury and $\mathrm{PH}$ in newborn rats. L-Citrulline may represent a novel therapeutic alternative to iNO for prevention of BPD. (Pediatr Res 68: 519-525, 2010)
\end{abstract}

$\mathrm{B}$ ronchopulmonary dysplasia (BPD), the chronic lung disease that follows acute respiratory failure after premature birth, is the most common complication in premature infants born $<28$ wk gestation, with an incidence of $30-50 \%$ (1). BPD currently lacks specific treatment or

Received March 3, 2010; accepted July 28, 2010.

Correspondence: Dr. Bernard Thébaud, M.D., Ph.D., Department of Pediatrics, University of Alberta, Women and Children's Health Research Institute, HMRC 407, Edmonton, AB, Canada T6G 2S2; e-mail: bthebaud@ualberta.ca

Supported by the Canadian Institutes of Health Research (CIHR), Alberta Heritage Foundation for Medical Research (AHFMR), Canada Foundation for Innovation (CFI), and a Canada Research Chair [B.T.] by the Maternal Fetal Neonatal Health Training Program (MFN) sponsored by CIHR-IHDCYH [A.V. and G.J.R.-P.]; by the AHFMR [G.J.R.-P.]; by NIH R01 HL75511 [J.L.A.]; and by NIH U54RR019453 [M.S.].

Current address [M.S.]: George Washington University, Children's National Medical Center, Washington, DC 20010.

Supplemental digital content is available for this article. Direct URL citations appear in the printed text and are provided in the HTML and PDF versions of this article on the journal's Web site (www.pedresearch.org). prevention strategies. BPD interrupts normal lung development and results in arrested alveolar and vascular growth $(2,3)$. The extent to which the disruption of lung growth leads to an earlier or more severe decline in respiratory function in later life is unknown (4). Case reports are emerging describing arrested alveolar growth in older children (5) and early onset emphysema in young adults (6) who were diagnosed with BPD.

Evidence suggests that NO promotes lung growth $(7,8)$. Lungs of endothelial NOS (eNOS)-deficient mice have a paucity of distal arteries and reduced alveolarization (9) and are more susceptible to disrupted lung growth after exposure to mild hypoxia and hyperoxia (8). Arrested alveolar growth in newborn rats after VEGF inhibition and in the chronically ventilated premature sheep and baboon models of BPD are associated with decreased lung eNOS protein and NO production. Inhaled NO (iNO) improves vascular and alveolar growth in these animal models (10-12). These data suggest that NO deficiency contributes to the decreased alveolarization in experimental BPD. Most recent randomized human trials suggest that iNO decreases the incidence of BPD in subsets of premature babies (13-15). Inconsistent efficacy, complexity of iNO delivery in nonintubated patients, and high cost provide rationales for efficacious alternatives to iNO.

Endogenous NO is produced from the metabolism of Larginine to L-citrulline, two amino acids generated by the urea cycle (16). L-Citrulline to L-arginine recycling in endothelial cells by the enzymes arginosuccinate synthetase (ASS) and arginosuccinate lyase (ASL) is proposed to be the principal mechanism for sustaining local L-arginine availability for eNOS-catalyzed NO production (17) (Fig. 1). Polymorphisms in the gene encoding carbamoylphosphate synthetase 1 , the mitochondrial enzyme catalyzing the rate-limiting step in hepatic L-citrulline formation via the urea cycle, influences NO metabolite concentrations and NO-mediated vasodilation

\footnotetext{
Abbreviations: ARG, arginase; ASL, arginosuccinate lyase; ASS, arginosuccinate synthetase; BPD, bronchopulmonary dysplasia; eNOS, endothelial NO synthase; iNO, inhaled nitric oxide; MWT, medial wall thickness; NOx, NO metabolites; P, postnatal day; PH, pulmonary hypertension; PPHN, persistent pulmonary hypertension of the newborn; RVH, right ventricular hypertrophy
} 


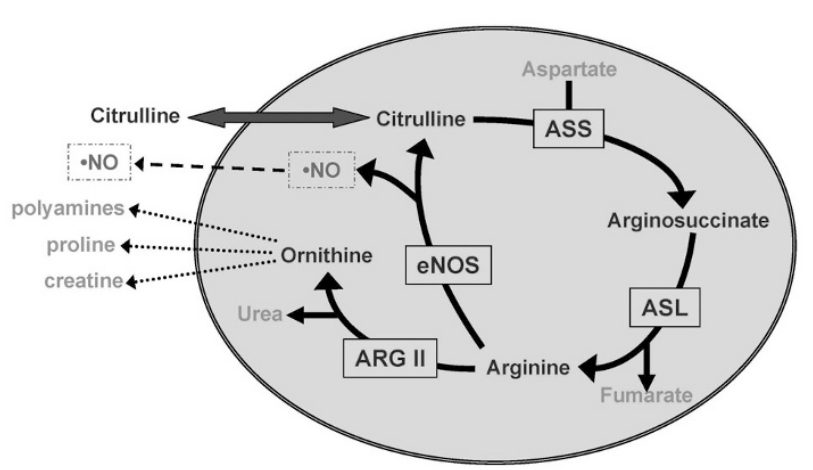

Figure 1. Schematic of the citrulline-arginine-NO pathway in endothelial cells. This figure depicts the intracellular enzymes that convert citrulline to arginine (ASS and ASL) and the enzymes eNOS that convert arginine to NO and citrulline and ARG II that converts arginine to ornithine and urea.

in humans (18). Infants with persistent pulmonary hypertension of the newborn (PPHN) or after bypass surgery for congenital heart disease have low plasma concentrations of arginine, citrulline, and NO metabolites (NOx) $(19,20)$, and this can be reversed by oral citrulline supplementation $(21,22)$. In addition to NO production, an adequate supply of precursor molecule is necessary to maintain the NOS complex in its "coupled" state (23). Uncoupling of NOS results in free radical oxygen production that may potentiate vascular damage and dysfunction (23). Thus, decreased concentrations of NO precursors and breakdown products suggest that inadequate NO production contributes to PPHN and abnormal vascular function. We hypothesized that L-citrulline supplementation preserves alveolar growth and prevents pulmonary hypertension $(\mathrm{PH})$ in a model of chronic oxygen-induced BPD in newborn rats.

\section{MATERIALS AND METHODS}

All procedures and protocols were approved by the Animal Health Care Committee of the University of Alberta. Expanded methods are available in the online as supplementary material (http://links.lww.com/PDR/A63).

Animal model. Experimental BPD was induced as previously described $(24,25)$. Sprague-Dawley rats (Charles River, Saint Constant, QC, Canada) were exposed to normoxia $\left(21 \% \mathrm{O}_{2} ; n=90\right)$ or hyperoxia $\left(95 \% \mathrm{O}_{2}\right.$, BPD model; $n=90$ ) from birth to postnatal day (P) 7, 10, or 14 in sealed Plexiglas chambers with continuous $\mathrm{O}_{2}$ monitoring (BioSpherix, Redfield, NY; supplementary material; http://links.lww.com/PDR/A63).

Experimental protocol. Newborn rat pups were randomized to four groups: one normoxia (21\%, control group; $n=45)$, two normoxia + L-citrulline $(n=45)$, three hyperoxia $\left(95 \% \mathrm{O}_{2}\right.$, BPD model; $\left.n=45\right)$, and four hyperoxia + L-citrulline $(n=45)$. L-Citrulline was administered daily from P4 to P14 via s.c. injection. The dose $\left(8 \mathrm{~g} / \mathrm{m}^{2} / \mathrm{d}\right)$ was based on the effective L-citrulline dosage reported in the literature (21). Controls received normal saline vehicle. From P14 to P21, rat pups were allowed to recover in $21 \%$ oxygen. Among animals housed in hyperoxia for $14 \mathrm{~d}, 26$ of the 30 untreated rats in the $\mathrm{O}_{2}$-induced $\mathrm{BPD}$ group survived; all 30 citrulline-treated rats in the $\mathrm{O}_{2}$-induced BPD group survived the study protocol.

Plasma amino acid levels. Amino acids (citrulline, arginine, and ornithine) were analyzed using a Hitachi 8800 (Hitachi USA) amino acid analyzer, and NOx were measured using a Sievers 280i instrument (GE Analytical Instruments, Boulder, CO) as previously described (21) (supplementary material; http://links.lww.com/PDR/A63).

Lung morphometry. fLungs were inflated and fixed via the trachea with a $4 \%$ formaldehyde solution at a constant pressure of $20 \mathrm{~cm} \mathrm{H}_{2} \mathrm{O}(24,25)$. Lungs were paraffin embedded, cut in 4- $\mu \mathrm{m}$ thick serial sections, and lung sections were stained with hematoxylin and eosin (H\&E). Alveolar structures were quantified using the mean linear intercept as described (25) (supplementary material; http://links.lww.com/PDR/A63).
Barium-gelatin angiograms. Barium was instilled into the pulmonary vasculature as previously described $(24,25)$ (supplementary material; $\mathrm{http} / / /$ links.lww.com/PDR/A63). Barium-filled pulmonary arteries were counted per high-powered field (100× magnification). Lungs of five animals/group, five sections/lung, and 10 high-power fields/section were counted.

Right ventricular hypertrophy and pulmonary artery remodeling. Right and left ventricles including the septum were weighed separately to determine the right ventricle $(\mathrm{RV})$ to left ventricle $(\mathrm{LV})+$ septum ratio $(\mathrm{S} ; \mathrm{RV} / \mathrm{LV}+\mathrm{S})$ as an index of right ventricular hypertrophy (RVH) (24). To assess pulmonary artery remodeling, the percent medial wall thickness (MWT) was calculated as $(2 \times$ wall thickness/external diameter $) \times 100 \%(24)$.

Western blot analysis. Whole rat lungs were used to determine eNOS, ASS, ASL, and arginase (ARG) II protein expression by Western blot using a modification of our previously published methods (26) (supplementary material; http://links.lww.com/PDR/A63).

$\boldsymbol{A R G}$ activity. ARG activity in frozen-lung tissue was measured by determining the amount of urea generated by the enzyme (27) (supplementary material; http://links.lww.com/PDR/A63).

Statistics. Values are expressed as the mean \pm SEM. Statistical comparisons were made with the use of ANOVA. Post hoc analysis used a Fisher's probable least significant difference test (Statview 5.1; Abacus Concepts). A value of $p<0.05$ was considered statistically significant.

\section{RESULTS}

Plasma concentrations of $N O$ precursors are decreased in $\mathrm{O}_{2}$-induced $\mathrm{BPD}$ in newborn rats. L-Citrulline and L-arginine plasma levels were significantly decreased in hyperoxicexposed animals on P7 compared with room air (RA) controls (Fig. $2 A$ inset and $B$ ). L-Citrulline supplementation significantly increased the plasma amino acids concentrations (Fig. $2 A-C)$. Plasma NOx levels were not significantly different between untreated RA and hyperoxic-exposed animals. LCitrulline supplementation significantly increased P7 plasma NOx levels in both normoxia- and hyperoxia-exposed rat pups (Fig. 2D).

${ }_{L}$-Citrulline prevents alveolar simplification in $\mathrm{O}_{2}$-induced $B P D$ in newborn rats. Hyperoxic-exposed rat pups displayed the characteristic features of alveolar simplification, with larger and fewer alveoli and decreased septation compared with normoxic animals (Fig. 3A). L-Citrulline preserved alveolar growth in hyperoxic rats as quantified by the mean linear intercept and septal counts (Fig. 3B) measured on P21. No effect of L-citrulline was observed in control rats.

${ }_{L}$-Citrulline preserves lung vascular growth in $\mathrm{O}_{2}$-induced $B P D$ in newborn rats. Hyperoxic-exposed rat pups showed a significant decrease in pulmonary arterial density compared with normoxic rat pups as demonstrated by barium angiograms performed on P21 (Fig. 4). Hyperoxia-exposed rat pups treated with L-citrulline exhibited increased arterial density when compared with untreated hyperoxic rat pups (Fig. 4). L-Citrulline had no effect on normoxic rats.

${ }_{L}$-Citrulline prevents $\mathrm{PH}$ in $\mathrm{O}_{2}$-induced $\mathrm{BPD}$ in newborn rats. Chronic exposure to hyperoxia for $14 \mathrm{~d}$ was associated with a significant increase in RVH (Fig. $5 A$ ) and \%MWT of small pulmonary arteries measured on P21 (Fig. 5B). LCitrulline attenuated these structural features of $\mathrm{PH}$ as indicated by a reduction in $\mathrm{RV} / \mathrm{LV}+\mathrm{S}$ and \%MWT (Fig. $5 A$ and $B$ ).

Hyperoxia alters the expression of proteins involved in the citrulline-arginine-NO cycle in a postnatal age-dependent manner. eNOS expression was not statistically different between control and hyperoxic rat lungs on P7 or P14 but was transiently increased on P10 in lungs from hyperoxia-exposed 

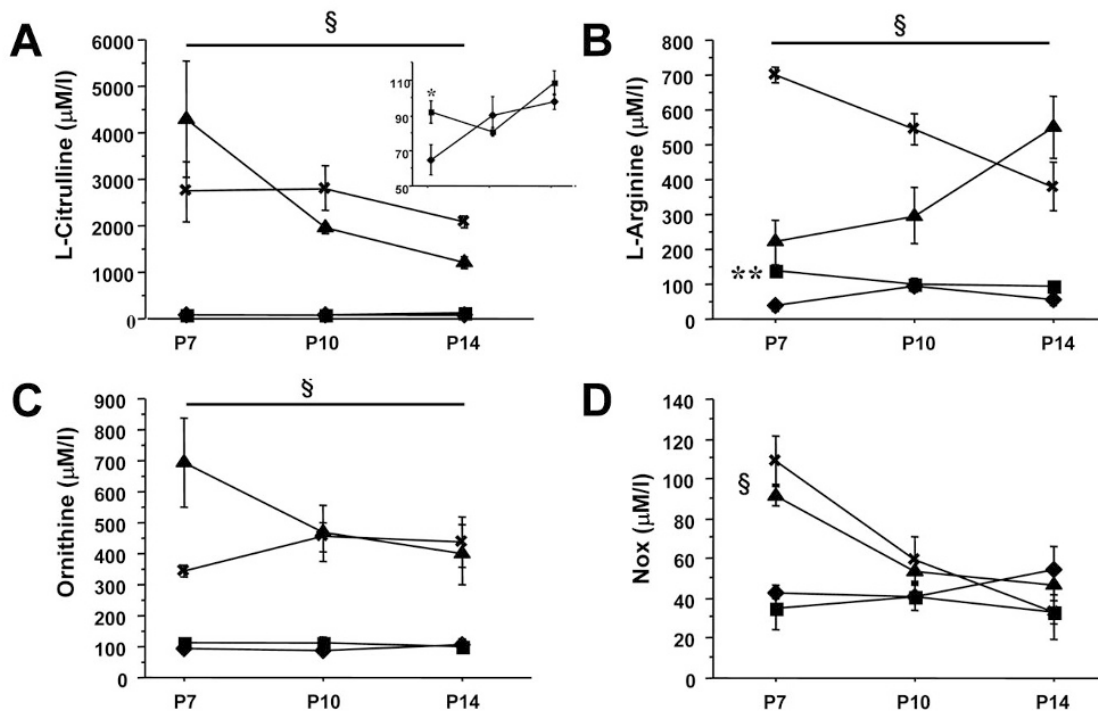

D

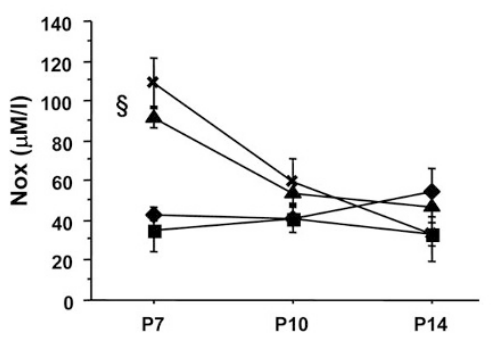

Figure 2. Effect of L-citrulline administration from P4 to 14 on amino acid plasma levels and NOx levels in normoxic control rats and $\mathrm{O}_{2-}$ induced BPD rats. Plasma levels of L-citrulline $(A)$, L-arginine $(B)$, ornithine $(C)$, and NOx $(D)$ were measured on P7, 10, and $14(n=5 /$ group, $* p<0.05$ and $* * p<0.0005$, normoxia $v s$ hyperoxia, $\S p<0.001$, normoxia (ם) and hyperoxia $(\bullet) v s$ normoxia + L-citrulline $(\boldsymbol{X})$ and hyperoxia + L-citrulline $(\boldsymbol{\Delta})$. Inset shows plasma levels of L-citrulline in normoxia (ם) and hyperoxia $(\diamond)$ only.
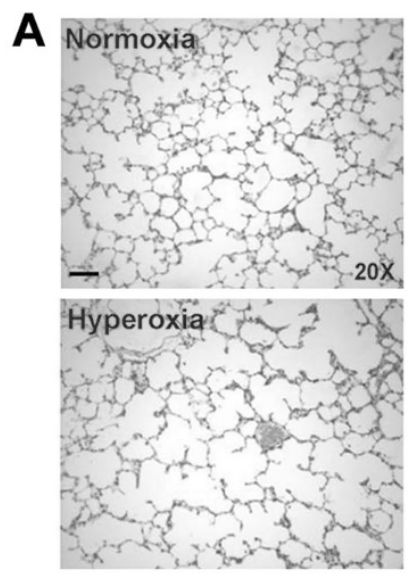
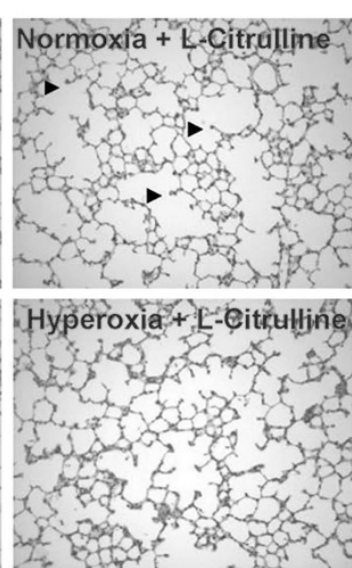

B

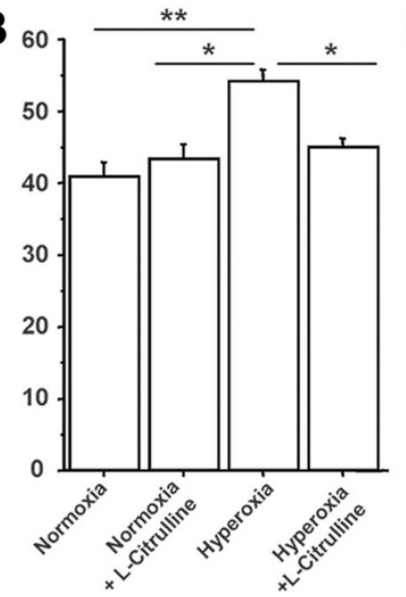

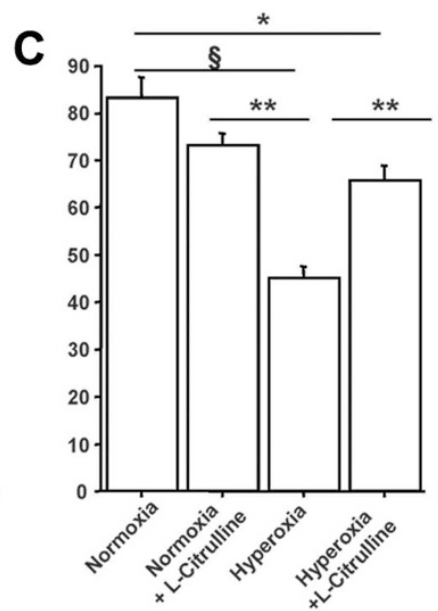

Figure 3. L-Citrulline prevents alveolar simplification in $\mathrm{O}_{2}$-induced $\mathrm{BPD}$ in newborn rats. Representative H\&E-stained lung sections show larger and fewer alveoli in hyperoxia-exposed lungs $(A)$, resulting in a significantly higher mean linear intercept $(\mathrm{Lm})(B)$ and lower septal counts than in control (normoxia) animals $(C)$. Treatment with L-citrulline preserved alveolar structure and significantly improved Lm and septal counts when compared with the experimental BPD model. $n=5 /$ group, $*_{*}^{*} p<0.001$ hyperoxia $v s$ normoxia, ${ }^{*} p<0.01$ hyperoxia $v s$ normoxia + L-citrulline and hyperoxia + L-citrulline for Lm. For septal counts, $\$ p<0.0001$ hyperoxia vs normoxia, ${ }^{* *} p<0.001$ hyperoxia $v s$ normoxia + L-citrulline and hyperoxia $v s$ L-citrulline, ${ }^{*} p<0.01$ normoxia $v s$ hyperoxia + L-citrulline.
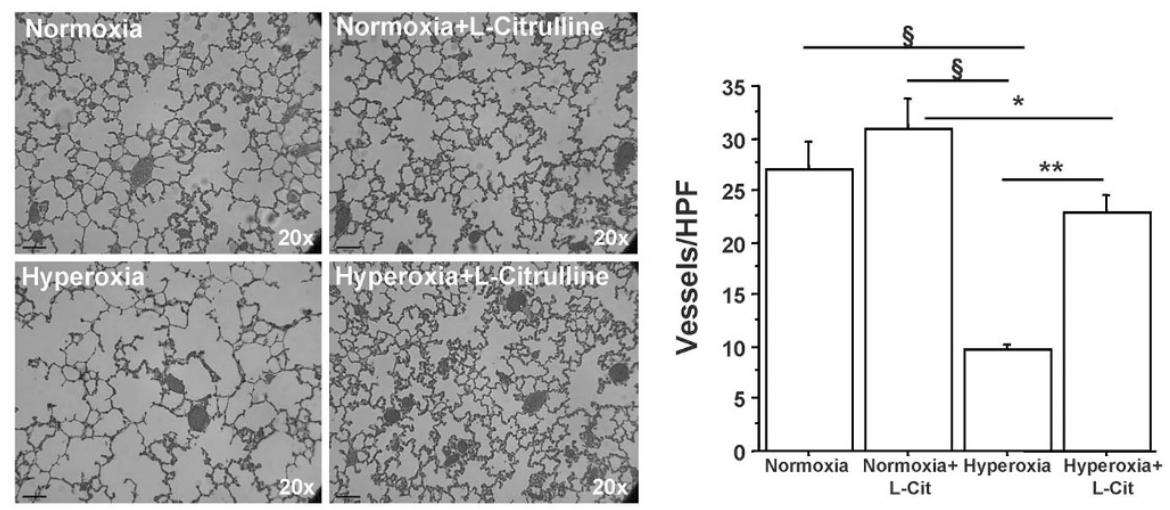

Figure 4. L-Citrulline improves lung angiogenesis in $\mathrm{O}_{2}$-induced BPD in newborn rats. Capillary density, as quantified by the number of barium-filled pulmonary arteries counted per high-power field, was significantly decreased in hyperoxia-exposed lungs compared with RA controls. Treatment with L-citrulline preserved lung capillary density in experimental BPD. $n=$ 5/group, $\$ p<0.0001$ hyperoxia $v s$ normoxia and normoxia + L-citrulline, ${ }^{* *} p<0.001$ hyperoxia $v s$ hyperoxia + L-citrulline, $* p<0.02$ normoxia + L-citrulline vs hyperoxia + Lcitrulline. rats. There was a significant increase in protein abundance of ASL throughout the $14 \mathrm{~d}$ exposure to hyperoxia; ASS protein expression was also significantly increased in hypoxic rat lungs on P7 and P10 but not on P14 (Fig. $6 A$ and $B$ ).
L-Citrulline increases protein expression of ASL in hyperoxia-exposed rat lungs. L-Citrulline treatment did not alter eNOS or ASS lung protein abundances in normoxia- or hyperoxia-exposed rat lungs on P7 or P10 (Fig. 7). However, 

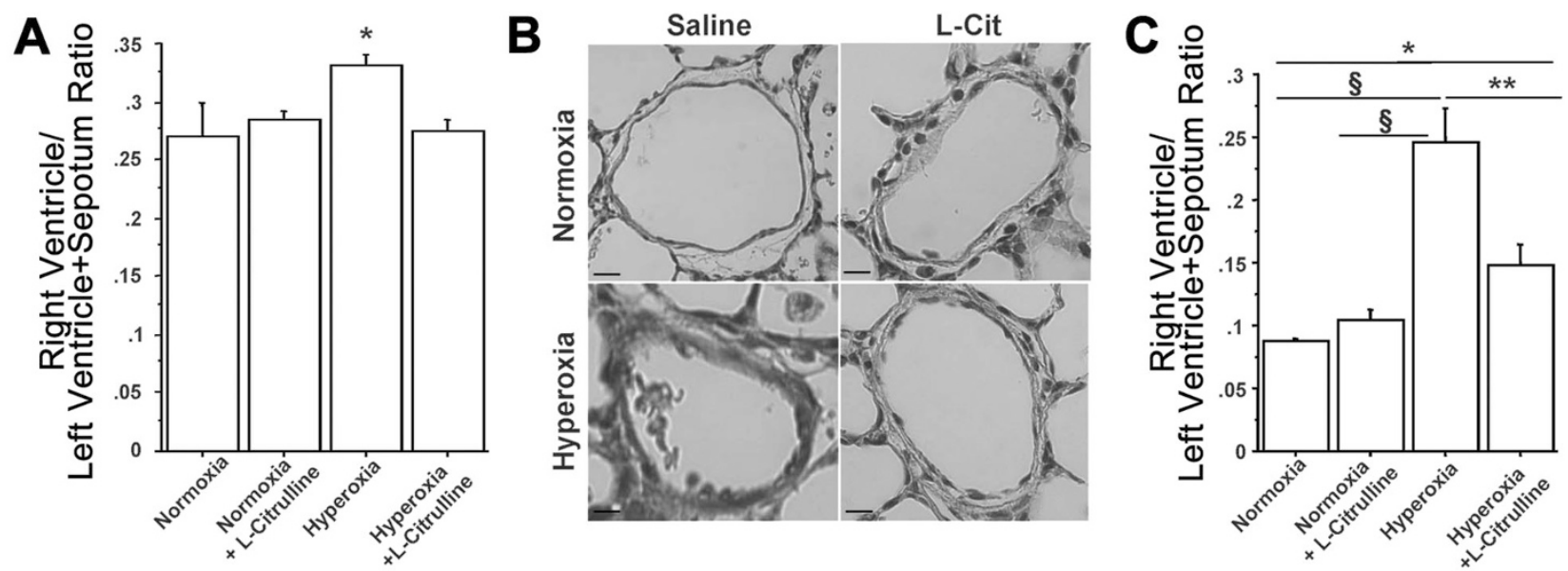

Figure 5. L-Citrulline prevents $\mathrm{PH}$ in $\mathrm{O}_{2}$-induced $\mathrm{BPD}$ in newborn rats. $A$, Hyperoxia-exposed rats had significant RVH as indicated by the increase in $\mathrm{RV} / \mathrm{LV}+\mathrm{S}$ ratio compared with normoxic controls. L-Citrulline reduced RVH. $n=5$ /group, $* p<0.05$ hyperoxia $v s$ other groups. $B$, Representative $\mathrm{H} \& \mathrm{E}$-stained lung sections showing pulmonary arteries displaying a thickened medial arterial wall in hyperoxic rat lungs compared with normoxic controls (scale bar is $65 \mu \mathrm{m}$, original magnification is $\times 40$ ). $C$, L-Citrulline significantly reduced the \%MWT compared with pulmonary arteries from untreated hyperoxic rat lungs. $n=5$ /group, $\$ p<0.0001$ hyperoxia $v s$ normoxia and normoxia + L-citrulline, $*^{*} p<0.002$ hyperoxia $v s$ hyperoxia + L-citrulline, ${ }^{*} p<0.05$ normoxia vs hyperoxia + L-citrulline.
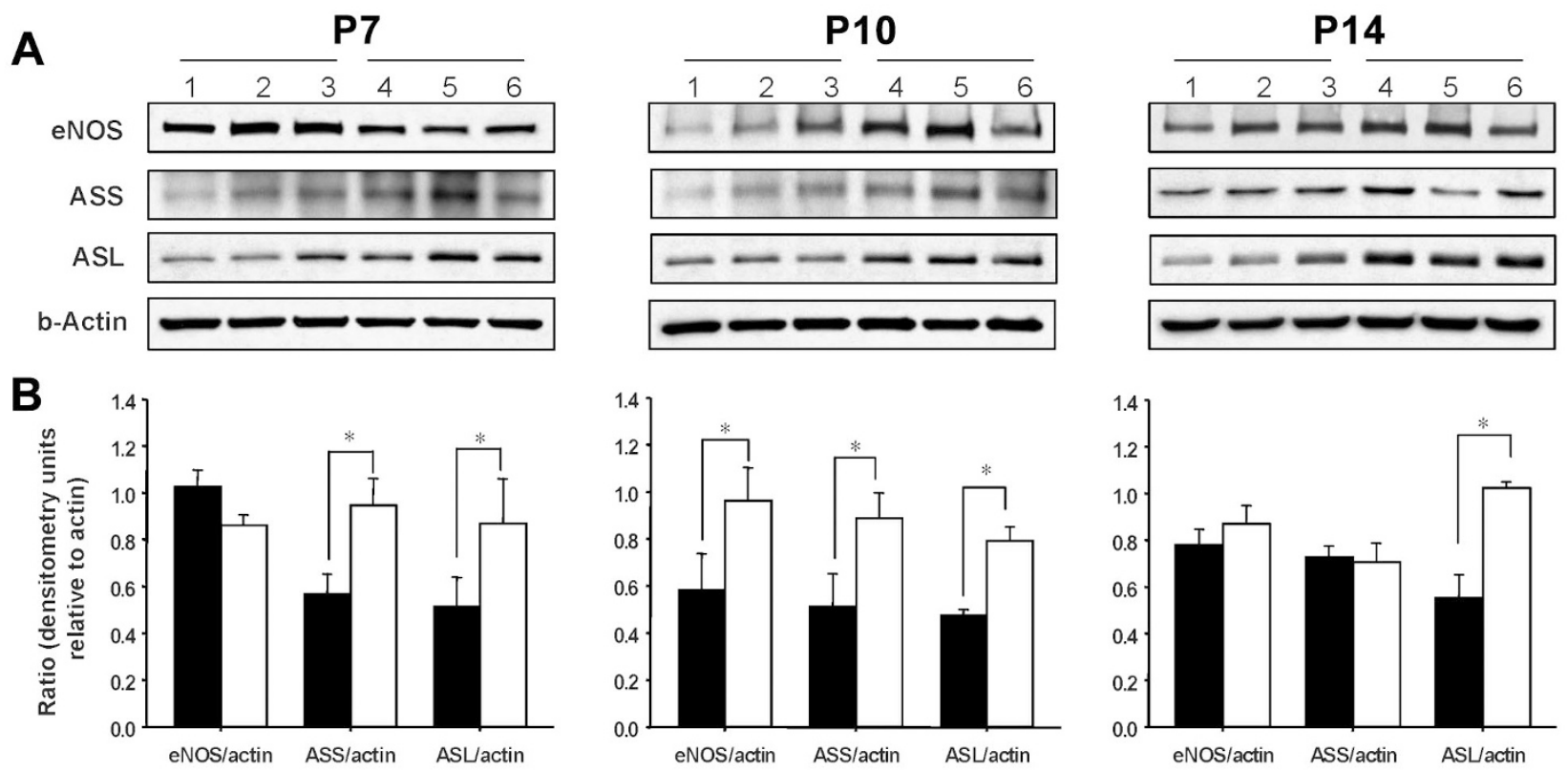

Figure 6. Effects of hyperoxia and postnatal age on protein abundance in rat lung homogenates. $A$, Representative Western blots demonstrating the abundance of eNOS, ASL, ASS, and $\beta$-actin in protein lysates from normoxic (lanes 1-3) and hyperoxic (lanes 4-6) rat lungs on P7, P10, and P14. Blots are representative of two separate studies performed on a total of $6-8$ normoxic and hyperoxic neonatal rat lungs at each postnatal time point. $B$, Summary densitometry data showing the effects of hyperoxia $(\square)$ on eNOS, ASS, and ASL protein expression relative to $\beta$-actin at each of three postnatal ages. * $p<0.05$ different from age-matched normoxic control (ם) group.

under hyperoxic conditions, L-citrulline increased ASL protein expression at both time points. Unexpectedly, L-citrulline treatment induced ARG II expression in normoxic and hyperoxic lungs on P7 and P10 (Fig. 7A-D).

Hyperoxia and L-citrulline treatment synergistically increase ARG II protein expression in newborn rat lungs. Chronic exposure to hyperoxia caused a significant increase in pulmonary ARG II expression (Fig. $8 A$ and $B$ ); the elevated ARG II protein abundance in hyperoxic lungs was further increased by L-citrulline therapy (Fig. $8 A$ and $B$ ).
Chronic exposure to hyperoxia was associated with a significant increase in lung ARG activity on P7 (Fig. 8C). L-Citrulline administration normalized lung ARG activity.

\section{DISCUSSION}

We demonstrate that $\mathrm{L}$-citrulline preserves alveolar and lung vascular development and prevents $\mathrm{PH}$ in an experimental $\mathrm{O}_{2}$-induced BPD model in newborn rats. Our results suggest a pivotal role for the $\mathrm{L}$-citrulline-NO pathway in the developing 

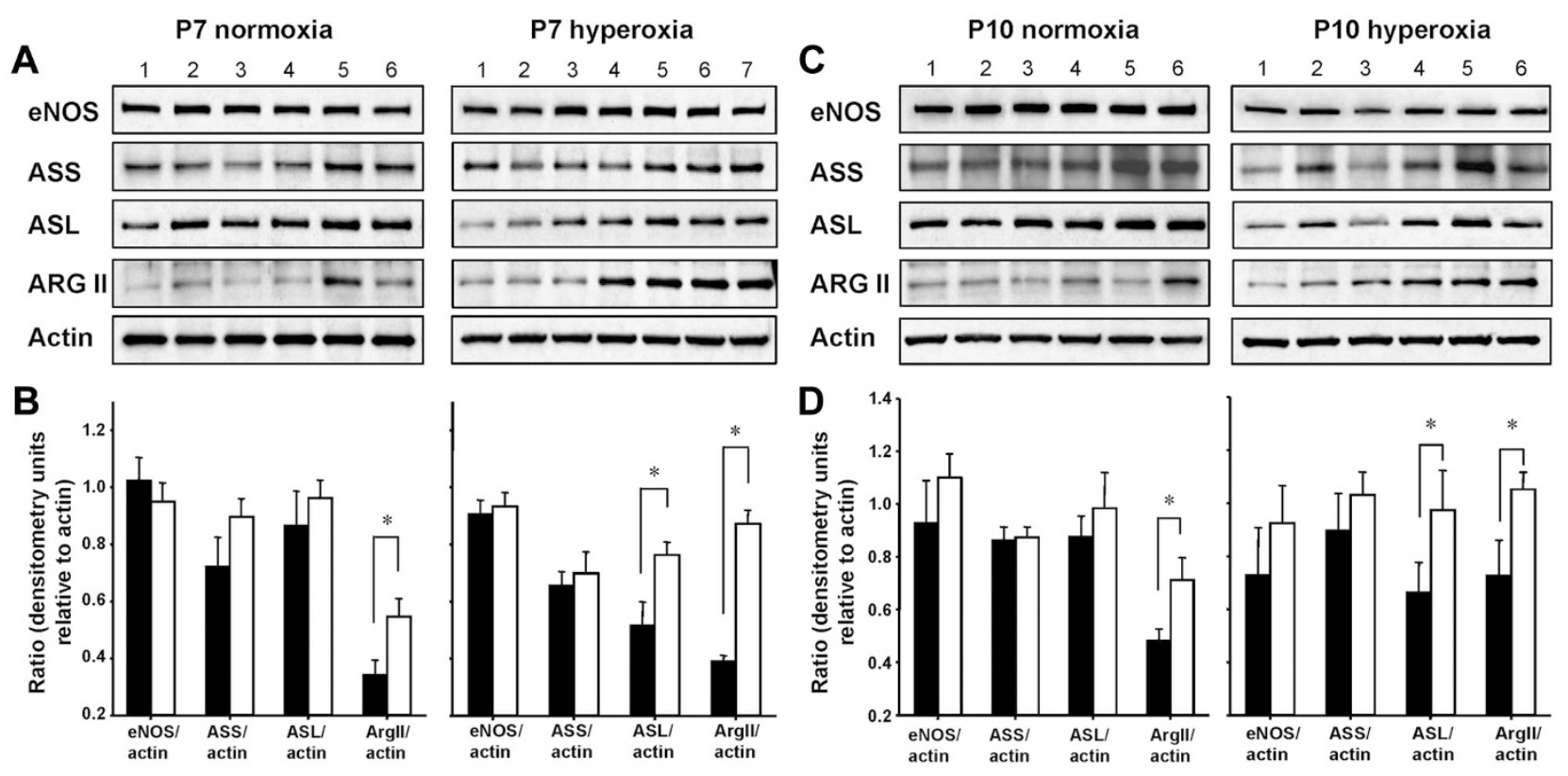

Figure 7. Effects of L-citrulline treatment on the abundance of proteins in the citrulline-arginine-NO regenerating pathway on P7 and P10. Representative Western blots demonstrating the abundance of eNOS, ASS, ASL, ARG II, and $\beta$-actin in protein lysates from L-citrulline-treated and untreated normoxic (left panel, lane 1-3: normoxia; lane 4-6: normoxia + L-citrulline) and hyperoxic (right panel, lane 1-3: hyperoxia; lane 4-7: hyperoxia + L-citrulline) rat lungs on P7 (A) and P10 (C). Blots are representative of two separate studies performed on a total of 6-8 untreated and citrulline-treated normoxic and hyperoxic neonatal rat lungs. Summary densitometry showing the effect of L-citrulline ( $\square$ ) on lung protein abundance of eNOS, ASS, ASL, and ARG II on P7 (B) and P10 $(D) . * p<0.05$ different from untreated normoxic or hyperoxic control (ם) group.
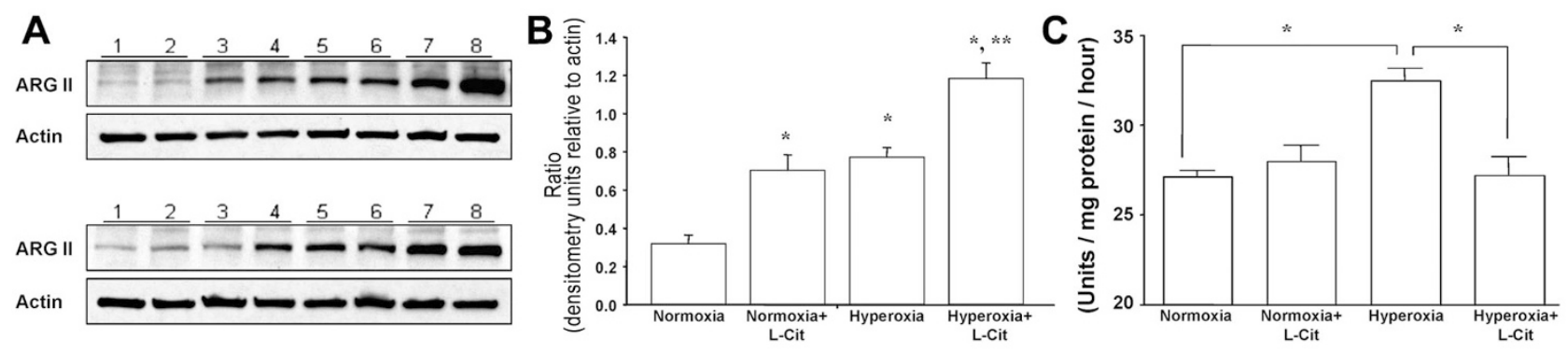

Figure 8. Effects of hyperoxia and L-citrulline treatment on protein abundance of ARG II and ARG activity in P7 rat lung homogenates. A, Representative Western blots of three separate studies performed on a total of six untreated (lane 1-2) and citrulline-treated (lane 3-4) normoxic and untreated (lane $5-6$ ) and citrulline-treated (lane 7-8) hyperoxic neonatal rat lungs. $B$, Summary densitometry showing that both hyperoxia and L-citrulline independently and synergistically increased ARG II expression in neonatal rat lungs. * $p<0.05$ different from normoxia and $* * p<0.05$ different from all other groups. C. L-Citrulline decreases pulmonary ARG activity in $\mathrm{O}_{2}$-induced BPD in newborn rats. Hyperoxic-exposed rats had significantly increased lung ARG activity on P7. L-Citrulline normalized lung ARG activity in hyperoxic-exposed rats. ARG activity was unchanged in L-citrulline-treated control rats. $n=$ 5/group, $* p<0.05$.

lung. This pathway may be an effective target to protect the lung from impaired alveolar development.

The availability of intracellular arginine is potentially a rate-limiting factor in the production of endogenous NO. Oral supplementation with L-arginine has been used in a variety of clinical conditions to improve NO-mediated vascular function (28). Experimental and human studies suggest that after oral administration, L-arginine is extensively metabolized by ARG in the gut wall and liver $(29,30)$. This may limit its bioavailability as a substrate for NOS and subsequent effects on vascular function. In addition, supplemental L-arginine enhances ARG expression and activity, thus reducing the effectiveness of L-arginine therapy. In contrast, L-citrulline is not metabolized in the intestine or liver and does not induce tissue ARG I, but rather inhibits its activity and is more effective in maintaining plasma $\mathrm{L}$-arginine concentrations than L-arginine itself in healthy volunteers (31). The physical relationship of the enzymes in cycling citrulline and arginine and producing NO (ASS, ASL, and eNOS) may also make L-citrulline a more effective extracellular supplement to improve NO production (32-35).

L-citrulline has no recognized toxicity and is used clinically as replacement therapy for children with certain types of urea cycle defects (36). Furthermore, oral L-citrulline, as a precursor to L-arginine and NO, improves sickle cell disease symptoms in children (37) and decreases PH after surgery for congenital heart disease $(21,22)$.

Our data suggest that plasma L-citrulline and L-arginine concentrations can be markedly increased by supplemental L-citrulline (Fig. 2). This limitation in metabolic precursors 
could contribute to low NO production and, thus, to arrested alveolar growth and $\mathrm{PH}$ in the $\mathrm{O}_{2}$-induced BPD model. Our data parallel findings in human infants with PPHN and in infants and children with $\mathrm{PH}$ after congenital heart disease surgery $(19,20)$. Our data also suggest that L-citrulline increases expression of ASL in the $\mathrm{O}_{2}$-injured lungs (Fig. 7). L-Citrulline exhibits good bioavailability with ease of movement across cellular membranes. The cytosolic portion of the urea cycle that includes ASS and ASL enables localized, intracellular production of L-arginine from citrulline within the pulmonary endothelium. This recycling pathway might be important in sustaining the production of $\mathrm{NO}$ in endothelial cells, especially when availability of L-arginine for NO synthesis becomes limiting. Although total eNOS protein expression did not change with citrulline treatment, the data suggest that eNOS function was enhanced. This is supported by the finding of increased NOx on $\mathrm{d} 7$ in normoxic and hyperoxic rats (Fig. 2D), which is a reflection of increased NOS activity and NO production. Although plasma levels of NOx were only transiently increased by L-citrulline treatment, local pulmonary production of NO may be inferred from the improved lung architecture and attenuated PH. L-citrulline's efficacy in this study mirrored those obtained with iNO in experimental BPD models (10-12).

PH contributes to morbidity and mortality in human BPD (38). The hyperoxic BPD model in rats also exhibits $\mathrm{PH}$ characterized by RVH and pulmonary artery medial wall thickening. L-citrulline attenuated RVH and pulmonary artery remodeling in this model (Figs. 4 and 5). These findings are consistent with a recent study of L-citrulline administration in a neonatal piglet model of chronic hypoxia-induced PH (39).

Among other mechanisms, increased ARG activity may account for abnormal airway and vascular remodeling in the hyperoxia model of BPD in newborn rats. ARGs mediate the conversion of L-arginine to urea and ornithine (40) (Fig. 1) and compete with NOS for L-arginine as substrate. Increased ARG activity in $\mathrm{PH}$ and other lung diseases, including asthma and cystic fibrosis, is believed to result in decreased availability of L-arginine for constitutive NOSs and consequently diminished NO production (41-43). Interestingly, lung ARG expression and activity in the rat is developmentally regulated and expressed at the highest levels in the fetus and newborn (44), suggesting that ARG contributes to the maintenance of high pulmonary vascular resistance during fetal life. Hyperoxia up-regulates ARG expression in the adult rat lung (45). Herein, we show that both ARG II expression and ARG activity are increased in chronic $\mathrm{O}_{2}$-exposed neonatal rat lungs (Figs. 7 and 8). This may limit arginine availability to NOS and contribute to decreased NO production in this model (46). Unexpectedly, we found that $\mathrm{L}$-citrulline increased expression of ARG II, the isoform expressed in nonhepatic tissue (Figs. 7 and 8). It had been previously reported that L-citrulline, unlike L-arginine, does not induce tissue ARG (31). Notably, and despite the increase in protein expression, L-citrulline normalized lung ARG activity in neonatal oxygen-induced lung injury (Fig. 8). An interesting link between increased ARG activity and BPD has been proposed recently. Ornithine, the downstream product of ARG activity, is further metabolized into polyamines that are involved in tissue repair and growth, and proline, the precursor for collagen formation (40) (Fig. 1). In bleomycin-induced lung fibrosis, ARG expression and activity are also increased $(47,48)$, suggesting that these enzymes contribute to lung remodeling and, thus, represent a therapeutic target.

In conclusion, this is the first report on the potential therapeutic benefit of L-citrulline in preventing $\mathrm{O}_{2}$-induced alveolar damage and altered angiogenesis in the developing lung. The bioavailability, safety, and efficacy of L-citrulline, combined with its low cost and ease of administration, make it an attractive therapeutic option that warrants further studies.

Acknowledgment. We thank Dr. Sowndramalingam Sankaralingam for technical assistance.

\section{REFERENCES}

1. Kinsella JP, Greenough A, Abman SH 2006 Bronchopulmonary dysplasia. Lancet 367:1421-1431

2. Stenmark KR, Abman SH 2005 Lung vascular development: implications for the pathogenesis of bronchopulmonary dysplasia. Annu Rev Physiol 67:623-661

3. Thébaud B, Abman SH 2007 Bronchopulmonary dysplasia: where have all the vessels gone? Roles of angiogenic growth factors in chronic lung disease. Am J Respir Crit Care Med 175:978-985

4. Stocks J, Coates A, Bush A 2007 Lung function in infants and young children with chronic lung disease of infancy: the next steps? Pediatr Pulmonol 42:3-9

5. Cutz E, Chiasson D 2008 Chronic lung disease after premature birth. N Engl J Med 358:743-745; author reply 745-746

6. Wong PM, Lees AN, Louw J, Lee FY, French N, Gain K, Murray CP, Wilson A, Chambers DC 2008 Emphysema in young adult survivors of moderate-to-severe bronchopulmonary dysplasia. Eur Respir J 32:321-328

7. Young SL, Evans K, Eu JP 2002 Nitric oxide modulates branching morphogenesis in fetal rat lung explants. Am J Physiol Lung Cell Mol Physiol 282:L379-L385

8. Balasubramaniam V, Tang JR, Maxey A, Plopper CG, Abman SH 2003 Mild hypoxia impairs alveolarization in the endothelial nitric oxide synthase-deficient mouse. Am J Physiol Lung Cell Mol Physiol 284:L964-L971

9. Han RN, Babaei S, Robb M, Lee T, Ridsdale R, Ackerley C, Post M, Stewart DJ 2004 Defective lung vascular development and fatal respiratory distress in endothelial NO synthase-deficient mice: a model of alveolar capillary dysplasia? Circ Res 94:1115-1123

10. Balasubramaniam V, Maxey AM, Morgan DB, Markham NE, Abman SH 2006 Inhaled NO restores lung structure in eNOS-deficient mice recovering from neonatal hypoxia. Am J Physiol Lung Cell Mol Physiol 291:L119-L127

11. Bland RD, Albertine KH, Carlton DP, MacRitchie AJ 2005 Inhaled nitric oxide effects on lung structure and function in chronically ventilated preterm lambs. Am J Respir Crit Care Med 172:899-906

12. McCurnin DC, Pierce RA, Chang LY, Gibson LL, Osborne-Lawrence S, Yoder BA, Kerecman JD, Albertine KH, Winter VT, Coalson JJ, Crapo JD, Grubb PH, Shaul PW 2005 Inhaled NO improves early pulmonary function and modifies lung growth and elastin deposition in a baboon model of neonatal chronic lung disease. Am J Physiol Lung Cell Mol Physiol 288:L450-L459

13. Ballard RA, Truog WE, Cnaan A, Martin RJ, Ballard PL, Merrill JD, Walsh MC, Durand DJ, Mayock DE, Eichenwald EC, Null DR, Hudak ML, Puri AR, Golombek SG, Courtney SE, Stewart DL, Welty SE, Phibbs RH, Hibbs AM, Luan X, Wadlinger SR, Asselin JM, Coburn CE 2006 Inhaled nitric oxide in preterm infants undergoing mechanical ventilation. N Engl J Med 355:343-353

14. Kinsella JP, Cutter GR, Walsh WF, Gerstmann DR, Bose CL, Hart C, Sekar KC, Auten RL, Bhutani VK, Gerdes JS, George TN, Southgate WM, Carriedo H, Couser RJ, Mammel MC, Hall DC, Pappagallo M, Sardesai S, Strain JD, Baier M, Abman SH 2006 Early inhaled nitric oxide therapy in premature newborns with respiratory failure. N Engl J Med 355:354-364

15. Schreiber MD, Gin-Mestan K, Marks JD, Huo D, Lee G, Srisuparp P 2003 Inhaled nitric oxide in premature infants with the respiratory distress syndrome. N Engl J Med 349:2099-2107

16. Moncada S, Higgs A 1993 The L-arginine-nitric oxide pathway. N Engl J Med 329:2002-2012

17. Goodwin BL, Solomonson LP, Eichler DC 2004 Argininosuccinate synthase expression is required to maintain nitric oxide production and cell viability in aortic endothelial cells. J Biol Chem 279:18353-18360

18. Summar ML, Gainer JV, Pretorius M, Malave H, Harris S, Hall LD, Weisberg A, Vaughan DE, Christman BW, Brown NJ 2004 Relationship between carbamoylphosphate synthetase genotype and systemic vascular function. Hypertension 43:186-191

19. Barr FE, Beverley H, VanHook K, Cermak E, Christian K, Drinkwater D, Dyer K, Raggio NT, Moore JH, Christman B, Summar M 2003 Effect of cardiopulmonary bypass on urea cycle intermediates and nitric oxide levels after congenital heart surgery. J Pediatr 142:26-30 
20. Pearson DL, Dawling S, Walsh WF, Haines JL, Christman BW, Bazyk A, Scott N, Summar ML 2001 Neonatal pulmonary hypertension-urea-cycle intermediates, nitric oxide production, and carbamoyl-phosphate synthetase function. N Engl J Med 344:1832-1838

21. Barr FE, Tirona RG, Taylor MB, Rice G, Arnold J, Cunningham G, Smith HA, Campbell A, Canter JA, Christian KG, Drinkwater DC, Scholl F, KavanaughMcHugh A, Summar ML 2007 Pharmacokinetics and safety of intravenously administered citrulline in children undergoing congenital heart surgery: potential therapy for postoperative pulmonary hypertension. J Thorac Cardiovasc Surg 134:319-326

22. Smith HA, Canter JA, Christian KG, Drinkwater DC, Scholl FG, Christman BW, Rice GD, Barr FE, Summar ML 2006 Nitric oxide precursors and congenital heart surgery: a randomized controlled trial of oral citrulline. J Thorac Cardiovasc Surg $132: 58-65$

23. Förstermann U, Munzel T 2006 Endothelial nitric oxide synthase in vascular disease: from marvel to menace. Circulation 113:1708-1714

24. Ladha F, Bonnet S, Eaton F, Hashimoto K, Korbutt G, Thebaud B 2005 Sildenafil improves alveolar growth and pulmonary hypertension in hyperoxia-induced lung injury. Am J Respir Crit Care Med 172:750-756

25. Thébaud B, Ladha F, Michelakis ED, Sawicka M, Thurston G, Eaton F, Hashimoto K, Harry G, Haromy A, Korbutt G, Archer SL 2005 Vascular endothelial growth factor gene therapy increases survival, promotes lung angiogenesis, and prevents alveolar damage in hyperoxia-induced lung injury: evidence that angiogenesis participates in alveolarization. Circulation 112:2477-2486

26. Aschner JL, Zeng H, Kaplowitz MR, Zhang Y, Slaughter JC, Fike CD 2009 Heat shock protein 90 -eNOS interactions mature with postnatal age in the pulmonary circulation of the piglet. Am J Physiol Lung Cell Mol Physiol 296:L555-L564

27. Corraliza IM, Campo ML, Soler G, Modolell M 1994 Determination of arginase activity in macrophages: a micromethod. J Immunol Methods 174:231-235

28. Böger RH 2008 L-Arginine therapy in cardiovascular pathologies: beneficial or dangerous? Curr Opin Clin Nutr Metab Care 11:55-61

29. Castillo L, deRojas TC, Chapman TE, Vogt J, Burke JF, Tannenbaum SR, Young VR 1993 Splanchnic metabolism of dietary arginine in relation to nitric oxide synthesis in normal adult man. Proc Natl Acad Sci U S A 90:193-197

30. Morris SM Jr. 2004 Enzymes of arginine metabolism. J Nutr 134:2743S-2747S

31. Romero MJ, Platt DH, Caldwell RB, Caldwell RW 2006 Therapeutic use of citrulline in cardiovascular disease. Cardiovasc Drug Rev 24:275-290

32. Flam BR, Hartmann PJ, Harrell-Booth M, Solomonson LP, Eichler DC 2001 Caveolar localization of arginine regeneration enzymes, argininosuccinate synthase and lyase, with endothelial nitric oxide synthase. Nitric Oxide 5:187-197

33. Isayama H, Nakamura H, Kanemaru H, Kobayashi K, Emson PC, Kawabuchi M, Tashiro N 1997 Distribution and co-localization of nitric oxide synthase and argininosuccinate synthetase in the cat hypothalamus. Arch Histol Cytol 60:477-492

34. Sullivan JC, Pollock JS 2003 NOS 3 subcellular localization in the regulation of nitric oxide production. Acta Physiol Scand 179:115-122
35. Yu JG, O’Brien WE, Lee TJ 1997 Morphologic evidence for L-citrulline conversion to $\mathrm{L}$-arginine via the argininosuccinate pathway in porcine cerebral perivascular nerves. J Cereb Blood Flow Metab 17:884-893

36. Tuchman M, Lee B, Lichter-Konecki U, Summar ML, Yudkoff M, Cederbaum SD, Kerr DS, Diaz GA, Seashore MR, Lee HS, McCarter RJ, Krischer JP, Batshaw ML 2008 Cross-sectional multicenter study of patients with urea cycle disorders in the United States. Mol Genet Metab 94:397-402

37. Waugh WH, Daeschner CW III, Files BA, McConnell ME, Strandjord SE 2001 Oral citrulline as arginine precursor may be beneficial in sickle cell disease: early phase two results. J Natl Med Assoc 93:363-371

38. Mourani PM, Sontag MK, Younoszai A, Ivy DD, Abman SH 2008 Clinical utility of echocardiography for the diagnosis and management of pulmonary vascular disease in young children with chronic lung disease. Pediatrics 121:317-325

39. Ananthakrishnan M, Barr FE, Summar ML, Smith HA, Kaplowitz M, Cunningham G, Magarik J, Zhang Y, Fike CD 2009 L-Citrulline ameliorates chronic hypoxiainduced pulmonary hypertension in newborn piglets. Am J Physiol Lung Cell Mol Physiol 297:L506-L511

40. Mori M, Gotoh T 2004 Arginine metabolic enzymes, nitric oxide and infection J Nutr 134:2820S-2825S

41. Grasemann H, Schwiertz R, Matthiesen S, Racke K, Ratjen F 2005 Increased arginase activity in cystic fibrosis airways. Am J Respir Crit Care Med 172:15231528

42. Morris CR, Kato GJ, Poljakovic M, Wang X, Blackwelder WC, Sachdev V, Hazen SL, Vichinsky EP, Morris SM Jr, Gladwin MT 2005 Dysregulated arginine metabolism, hemolysis-associated pulmonary hypertension, and mortality in sickle cell disease. JAMA 294:81-90

43. Morris CR, Poljakovic M, Lavrisha L, Machado L, Kuypers FA, Morris SM Jr 2004 Decreased arginine bioavailability and increased serum arginase activity in asthma. Am J Respir Crit Care Med 170:148-153

44. Belik J, Shehnaz D, Pan J, Grasemann H 2008 Developmental changes in arginase expression and activity in the lung. Am J Physiol Lung Cell Mol Physiol 294:L498 L504

45. Que LG, Kantrow SP, Jenkinson CP, Piantadosi CA, Huang YC 1998 Induction of arginase isoforms in the lung during hyperoxia. Am J Physiol 275:L96-L102

46. Belik J, Jankov RP, Pan J, Yi M, Chaudhry I, Tanswell AK 2004 Chronic O2 exposure in the newborn rat results in decreased pulmonary arterial nitric oxide release and altered smooth muscle response to isoprostane. J Appl Physiol 96:725730

47. Endo M, Oyadomari S, Terasaki Y, Takeya M, Suga M, Mori M, Gotoh T 2003 Induction of arginase I and II in bleomycin-induced fibrosis of mouse lung. Am J Physiol Lung Cell Mol Physiol 285:L313-L321

48. Kitowska K, Zakrzewicz D, Konigshoff M, Chrobak I, Grimminger F, Seeger W, Bulau P, Eickelberg O 2008 Functional role and species-specific contribution of arginases in pulmonary fibrosis. Am J Physiol Lung Cell Mol Physiol 294:L34-L45 